\title{
Socioeconomic inequalities in psychological distress and suicidal behaviours among Indigenous peoples living off-reserve in Canada
}

\author{
Mohammad Hajizadeh PhD, Amy Bombay PhD, Yukiko Asada PhD
}

Cite as: CMAJ 2019 March 25;191:E325-36. doi: 10.1503/cmaj.181374

Visual abstract available at www.cmaj.ca/lookup/suppl/doi:10.1503/cmaj.181374/-/DC2

\begin{abstract}
BACKGROUND: Indigenous peoples in Canada have high rates of psychological distress and suicide. We sought to assess the socioeconomic inequalities in psychological distress and suicidal behaviours, and the factors that explain them within Indigenous peoples living off-reserve.
\end{abstract}

METHODS: Using the nationally representative 2012 Aboriginal Peoples Survey collected from Indigenous adults living off-reserve in Canada, we measured income-related inequalities in psychological distress (measured on the 10-item Kessler Psychological Distress Scale) and suicidal behaviours (suicidal ideation and suicide attempt) and identified factors contributing to these inequalities using the concentration index $(C)$ approach.

RESULTS: Among 14410 individuals representing 600750 Indigenous adults (aged $\geq 18 \mathrm{yr}$ ) living off-reserve in Canada, the mean score of psychological distress was $16.1 ; 19.4 \%$ reported lifetime suicidal ideation and $2.2 \%$ reported a lifetime suicide attempt. Women had higher psychological distress scores (mean score $16.7 \mathrm{v}$. $15.2, p<0.001$ ), and prevalence of suicidal ideation ( $21.9 \%$ v. $16.1 \%, p<0.001)$ and suicide attempts $(2.3 \%$ v. $2.0 \%, p=$ 0.002 ) than men. Poorer individuals disproportionately experienced higher psychological distress ( $C=-0.054,95 \%$ confidence interval $[\mathrm{Cl}]-0.057$ to -0.050 ), suicidal ideation $\left(C_{n}=-0.218,95 \% \mathrm{Cl}\right.$ -0.242 to -0.194$)$ and suicide attempts $\left(C_{n}=-0.327,95 \% \mathrm{Cl}-0.391\right.$ to -0.263$)$. Food insecurity and income, respectively, accounted for $40.2 \%$ and $13.7 \%$ of the psychological distress, $26.7 \%$ and $18.2 \%$ of the suicidal ideation and $13.4 \%$ and $7.8 \%$ of the suicide attempts concentrated among low-income Indigenous peoples.

INTERPRETATION: Substantial incomerelated inequalities in psychological distress and suicidal behaviours exist among Indigenous peoples living offreserve in Canada. Policies designed to address major contributing factors such as food insecurity and income may help reduce these inequalities.
W orldwide, Indigenous populations have high rates of suicide and psychological distress, the latter characterized by psychological and physiologic symptoms of anxiety and depression..$^{1-3}$ Suicide is a major cause of death among First Nations, Métis and Inuit peoples, ${ }^{4}$ the 3 distinct Indigenous groups in Canada. Suicide rates among Indigenous peoples, when considered collectively, are 2 to 3 times higher than among non-Indigenous Canadians. ${ }^{5}$ Rates of suicide and distress vary considerably across the Indigenous groups. During the period between 1991 and 2001, the Indigenous to non-Indigenous suicide mortality rate ratio was 1.60 for Métis men, 0.85 for Métis women, 1.66 for status First Nations men and 1.86 for status First Nations women. ${ }^{6}$ Suicide rates among the Inuit, which are among the highest in the world, are up to 10 times higher than the overall rate for Canada. ${ }^{5,7,8}$ Suicide led to life expectancy losses of
4.8 years for men and 1.2 years for women in Inuit Nunangat in 1999-2003. ${ }^{9}$ Suicide rates are higher among First Nations peoples living on-reserve than among Indigenous peoples living offreserve. ${ }^{10}$ The rate ratios for potential years of life lost owing to suicide among status First Nation men living on- and off-reserve compared with non-Indigenous men (women) were 2.88 (3.71) and 1.11 (0.76), respectively. ${ }^{11}$ Furthermore, except for Métis men, suicidal thoughts among Indigenous peoples are more common than among non-Indigenous Canadians. ${ }^{12}$

Historical and ongoing experiences associated with colonization accompanied by inequities in income, employment opportunities, housing and food security, among other factors, have resulted in pervasive health problems among Indigenous peoples.,13-21 Indigenous populations have the poorest health outcomes in Canada, often similar to those of populations in developing countries..$^{18}$ 
Income has been shown to be a main determinant of health among both Indigenous ${ }^{22}$ and the general Canadian populations. ${ }^{23}$ Recent evidence points to a particularly strong incomerelated gradient in mental health outcomes compared with most other health outcomes. ${ }^{24}$ The role of income may be especially important given persistently lower incomes among Indigenous populations compared with non-Indigenous populations, with a gap of $25 \%$ in 2015 and little improvement since $2005 .{ }^{25}$ There is scant literature on socioeconomic inequalities in mental health within Indigenous peoples in Canada, despite considerable variations in collective histories, present-day circumstances and cultures.

Using data from the 2012 Aboriginal Peoples Survey, we quantified the extent of and factors accounting for incomerelated inequalities in psychological distress and suicidal behaviours among status First Nations, non-status First Nations, Métis and Inuit peoples living off-reserve in Canada.

\section{Methods}

\section{Sample}

We used data from the confidential master files of the 2012 Aboriginal Peoples Survey conducted by Statistics Canada. A large cross-sectional survey, with a response rate in 2012 of $76 \%,{ }^{26}$ the Aboriginal Peoples Survey collects detailed data on the social and economic conditions (e.g., education, employment, health, language, income, housing and mobility) from Indigenous peoples aged 6 years and older who are living in private dwellings, excluding those living on Indian settlements and reserves and in specific First Nations communities in the Northwest Territories and Yukon. ${ }^{26}$ Data from survey participants were adjusted to represent Indigenous adults living off-reserve in Canada using bootstrapped weights, as per guidelines from Statistics Canada's Research Data Centres. ${ }^{27}$

\section{Variables}

\section{Mental health outcome variables}

The outcome variables included psychological distress, lifetime suicidal ideation and lifetime suicide attempt. The 10-item Kessler Psychological Distress Scale (K10) (Appendix 1A, available at www.cmaj.ca/lookup/suppl/doi:10.1503/cmaj.181374/-/DC1) ${ }^{28}$ was used to measure nonspecific psychological distress, composed of items assessing psychological and physiologic symptoms of anxiety and depression. Total scores of the K10 range from 10 (no distress) to 50 (severe distress). Studies have shown that the $\mathrm{K} 10$ is psychometrically valid and appropriate for use in Indigenous populations living off-reserve in Canada. ${ }^{28-31}$ The Aboriginal Peoples Survey also asked questions related to suicidal behaviours, from which we created 2 binary variables of suicidal behaviour: lifetime suicidal ideation and suicide attempt.

\section{Socioeconomic position variables}

We used the natural log of equivalized household income (i.e., household income divided by the square root of household size) $)^{32}$ as a proxy of socioeconomic status.

\section{Explanatory variables}

The decomposition analysis included variables known to be associated with mental health outcomes in Indigenous populations ${ }^{33-41}$ and available in the Aboriginal Peoples Survey. These included 3 demographic variables (sex [male, female], age [continuous] and marital status [single, married, divorced or widowed]), and cultural group (status First Nations, non-status First Nations, Métis, Inuit); 6 socioeconomic variables (natural log of equivalized household income, education [ $\leq$ grade 8 , grades 9-10, grade 11-secondary completed, some postsecondary, postsecondary degree/diploma], employment status [employed, not in the labour force, unemployed], household crowding [rooms per capita $\leq 2,>2$ to $4,>4$ ]; housing maintenance [regular, minor or major maintenance needed]; food security [high, low, very low]); 2 behavioural variables (regular drinker, daily tobacco use); 2 social connectedness variables (strong extended family ties, no one to turn to for support); 4 cultural engagement and language variables (clothing or footwear; art craft, hunting, fishing or trapping; plant gathering; speaking Indigenous language); and 2 geographic variables (urbanicity [rural, small population centre, medium population centre, urban], region [Atlantic provinces, Quebec, Ontario, Manitoba, Saskatchewan, Alberta, British Columbia, territories]). Appendix $1 \mathrm{~B}$ reports the definitions and descriptive statistics of the variables used in the study.

\section{Statistical analysis}

The concentration index $(C)^{42}$ was used to quantify incomerelated inequalities in mental health outcomes. The $C$ is computed based on the concentration curve, which depicts the cumulative percentage of the population ranked by ascending order of a socioeconomic status variable (e.g., income), on its x-axis, against the cumulative percentage of a health outcome (e.g., psychological distress score). The $C$ is calculated as twice the area between the diagonal (the perfect equality line) and the concentration curve. ${ }^{43}$ The value of $C$ varies from -1 to 1 , with 0 indicating perfect equality. ${ }^{44}$ Because 2 of our outcome variables were binary (lifetime suicide ideation and attempt), the normalized concentration index $\left(C_{n}\right)$ was used for these outcomes. ${ }^{45} \mathrm{~A}$ negative (positive) value of the $C$ and $C_{n}$ indicate that the health outcome is disproportionately concentrated among individuals with low (high) socioeconomic status.

The $C$ and $C_{n}$ were decomposed to identify the contributions of a set of $k$ explanatory variables to income-related inequality in each of the health-outcome variables. The decomposition of the $C$ and $C_{n}$ is based on the regression analysis of the association between the outcome variable and a set of $k$ explanatory variables. ${ }^{46}$ The negative contribution of a factor to the $C_{n}$ suggests that income-related distribution of the factor (i.e., the $C_{k}$ ) and the association between the factor and health outcomes increase the concentration of health outcome among the poor (a positive contribution would increase the concentration of the health outcome among the rich). ${ }^{44,46}$ Appendix $1 C$ provides a detailed description of the $C$ and $C_{n}$ and the decomposition of these 2 indexes. The $F$ and $\chi^{2}$ statistics were used to test the differences among covariates for continuous (i.e., psychological distress) and categorical (i.e., 
suicidal ideation and suicide attempt) variables, respectively. We used bootstrap weights provided by Statistics Canada in the analyses to obtain estimates that are representative of Indigenous populations living off-reserve in Canada and to take into account the complex survey design using the SVY command ${ }^{47}$ in Stata $14 .{ }^{48}$ We considered $p<0.05$ statistically significant.

\section{Ethics approval}

We accessed the Aboriginal Peoples Survey through Statistics Canada's Atlantic Research Data Centre. Data accessed through the Research Data Centres, which follow strict disclosure protocols according to the Statistics Act, are exempt from research ethics board review based on the Tri-Council Policy Statement: Ethical Conduct for Research Involving Humans (TCPS2) article 2.2 (a).

\section{Results}

\section{Prevalence of psychological distress and suicidal behaviours}

The Aboriginal Peoples Survey included information on 28410 respondents. After we excluded 10550 individuals aged less than 18 years and with multiple Indigenous identities, and 3450 missing values in outcome or explanatory variables, our final sample consisted of 14410 individuals, representing 600750 Indigenous people living off-reserve in Canada (Table 1).

The mean distress score among Indigenous peoples was 16.1 for both sexes, 15.2 for men and 16.7 for women $(p<0.001)$. The prevalence rates of lifetime suicidal ideation and suicide attempt among Indigenous peoples in 2012 for both sexes were $19.4 \%$ (men: 16.1\%; women: $21.9 \%, p<0.001$ ) and 2.2\% (men: $2.0 \%$; women: $2.3 \%, p=0.002$ ), respectively (Tables 2 and 3).

The mean distress score varied $(p<0.001)$ among the 4 Indigenous cultural groups, with Inuit having the lowest mean distress score (total: 15.4; men: 14.6; women: 16.0) and non-status First Nations having the highest mean distress score (total: 16.8; men: 15.6; women: 17.7) (Table 2). There was also a significant variation in the prevalence of suicidal ideation $(p<0.001)$ and suicide attempt $(p<0.001)$ among the 4 Indigenous cultural groups, with Métis having the lowest rates of suicidal ideation (total: $17.5 \%$; men: $13.8 \%$; women: $20.5 \%$ ) and suicide attempt (total: $1.7 \%$; men: $1.2 \%$; women: $2.2 \%$ ), and Inuit having the highest rates of suicidal ideation (total: $22.7 \%$; men: $20.7 \%$; women: $24.3 \%$ ) and suicide attempt (total: $3.2 \%$; men: $1.6 \%$; women: $4.5 \%)$. The prevalence of lifetime suicide attempt was not different between Métis and status and non-status First Nations women (Table 3).

\section{Income-related inequalities in psychological distress} and suicidal behaviours

The negative values of the $C$ for psychological distress $(-0.054$, $95 \%$ confidence interval $[\mathrm{Cl}]-0.057$ to -0.050$)$ and the $C_{n}$ for lifetime suicidal ideation $(-0.218,95 \% \mathrm{Cl}-0.242$ to -0.194$)$ and lifetime suicide attempt $(-0.327,95 \% \mathrm{Cl}-0.391$ to -0.263$)$ suggest that these mental health outcomes are disproportionately concentrated among those with lower income. Income-related inequality in psychological distress was marginally greater among women $(C=-0.055,95 \% \mathrm{Cl}-0.060$ to -0.050$)$ than men ( $C=-0.046,95 \% \mathrm{Cl}-0.051$ to -0.040$)$, whereas income-related inequalities in suicide attempts were higher among men $\left(C_{n}=\right.$ $-0.462,95 \% \mathrm{Cl}-0.562$ to -0.362$)$ than women $\left(C_{n}=-0.236,95 \%\right.$ $\mathrm{Cl}-0.320$ to -0.152 ). For both men and women, income-related inequality in psychological distress was highest among status First Nations $(C=-0.069,95 \% \mathrm{Cl}-0.082$ to -0.057$)$ and lowest among Inuit $(C=-0.039,95 \% \mathrm{Cl}-0.048$ to -0.030$)$. Status First Nations men had the highest income-related inequalities in lifetime suicidal ideation $\left(C_{n}=-0.378,95 \% \mathrm{Cl}-0.496\right.$ to -0.260$)$ and suicide attempt $\left(C_{n}=-0.629,95 \% \mathrm{Cl}-0.868\right.$ to -0.390$)$ among Indigenous male cultural groups. Among women, incomerelated inequalities in suicidal behaviours were highest among Métis (suicidal ideation: $C_{n}=-0.215,95 \% \mathrm{Cl}-0.264$ to -0.166 ; suicide attempt: $C_{n}=-0.269,95 \% \mathrm{Cl}-0.407$ to -0.131 ) and lowest among Inuit (suicidal ideation: $C_{n}=-0.153,95 \% \mathrm{Cl}-0.226$ to -0.080 ; suicide attempt: $C_{n}=-0.126,95 \% \mathrm{Cl}-0.278$ to -0.026 ) (Table 4).

\section{Determinants of income-related inequalities in psychological distress and suicidal behaviours} The decomposition analyses showed food insecurity as the most important factor contributing to income-related inequalities in the 3 mental health outcomes among men and women (Table 5). Food insecurity accounted for $40.2 \%$ (men: $38.4 \%$; women: 42.9\%), 26.7\% (men: $21.4 \%$; women: $32.2 \%$ ) and $13.4 \%$ (men:

Table 1: Weighted and unweighted number of observations by sex and cultural group*

Total

\section{Unweighted}

14410
Male

Unweighted

6510

\section{Weighted}

260510

\section{Unweighted}

7900
Female

Cultural group

\section{Status First Nations}

Non-status First Nations

Métis

Inuit
4630

1460

5970

2360
205940

96440

271990

26380
1990

640

2800

1080
84200

41540

123380

11400
2630

820

3160

1290
Weighted

340240
*The number of observations was rounded to base 10 as per the data disclosure policy of Statistics Canada's Research Data Centres for the Aboriginal Peoples Survey. 
Table 2 (part 1 of 2): Mean scores* on the 10-item Kessler Psychological Distress Scale among Indigenous peoples living

off-reserve $(n=14410)$

\begin{tabular}{|c|c|c|c|c|c|c|}
\hline Characteristic & \multicolumn{2}{|c|}{ Total } & \multicolumn{2}{|c|}{ Men } & \multicolumn{2}{|c|}{ Women } \\
\hline \multicolumn{7}{|l|}{ Demographic variable } \\
\hline \multicolumn{7}{|l|}{ Sex } \\
\hline \multicolumn{7}{|l|}{ Marital status } \\
\hline Married & 17.0 & $<0.001$ & 16.3 & $<0.001$ & 17.6 & $<0.001$ \\
\hline Divorced or widowed & 15.2 & & 14.5 & & 15.8 & \\
\hline Single & 17.3 & & 15.7 & & 17.8 & \\
\hline Non-status First Nations & 16.8 & & 15.6 & & 17.7 & \\
\hline Inuit & 15.4 & & 14.6 & & 16.0 & \\
\hline \multicolumn{7}{|l|}{ Socioeconomic variable } \\
\hline \multicolumn{7}{|l|}{ Education level } \\
\hline$\leq$ Grade 8 & 16.5 & $<0.001$ & 16.0 & $<0.001$ & 17.0 & $<0.001$ \\
\hline Grades 9-10 & 17.9 & & 16.2 & & 19.3 & \\
\hline Grade 11 -secondary completed & 16.2 & & 15.3 & & 16.9 & \\
\hline Some postsecondary & 16.4 & & 15.5 & & 17.3 & \\
\hline Postsecondary degree/diploma & 15.4 & & 14.6 & & 15.9 & \\
\hline Rooms per capita $>2$ to 4 & 15.5 & & 14.7 & & 16.1 & \\
\hline Rooms per capita $>4$ & 15.1 & & 14.7 & & 15.5 & \\
\hline \multicolumn{7}{|l|}{ Housing maintenance } \\
\hline Regular & 15.5 & $<0.001$ & 14.8 & $<0.001$ & 16.0 & $<0.001$ \\
\hline Minor & 16.3 & & 15.3 & & 17.1 & \\
\hline Major & 17.9 & & 16.6 & & 18.8 & \\
\hline \multicolumn{7}{|l|}{ Food security } \\
\hline High & 14.9 & $<0.001$ & 14.3 & $<0.001$ & 15.4 & $<0.001$ \\
\hline Low & 18.8 & & 18.2 & & 19.3 & \\
\hline Very low & 23.7 & & 22.2 & & 24.4 & \\
\hline \multicolumn{7}{|l|}{ Behavioural variable } \\
\hline \multicolumn{7}{|l|}{ Drinking habit } \\
\hline Regular drinker (yes) & 15.7 & $<0.001$ & 14.9 & $<0.001$ & 16.4 & $<0.001$ \\
\hline Regular drinker (no) & 16.6 & & 15.7 & & 17.1 & \\
\hline \multicolumn{7}{|l|}{ Smoking habit } \\
\hline Daily tobacco use (yes) & 17.9 & $<0.001$ & 16.8 & $<0.001$ & 18.7 & $<0.001$ \\
\hline Daily tobacco use (no) & 15.3 & & 14.4 & & 15.9 & \\
\hline
\end{tabular}


Table 2 (part 2 of 2): Mean scores ${ }^{\star}$ on the 10-item Kessler Psychological Distress Scale among Indigenous peoples living off-reserve $(n=14410)$

\begin{tabular}{|c|c|c|c|c|c|c|}
\hline \multirow[b]{2}{*}{ Characteristic } & \multicolumn{2}{|c|}{ Total } & \multicolumn{2}{|c|}{ Men } & \multicolumn{2}{|c|}{ Women } \\
\hline & Mean score & $p$ value & Mean score & $p$ value & Mean score & $p$ value \\
\hline \multicolumn{7}{|l|}{ Social connectedness variable } \\
\hline Strong extended family tie (yes) & 15.1 & $<0.001$ & 14.1 & $<0.001$ & 15.8 & $<0.001$ \\
\hline Strong extended family tie (no) & 17.0 & & 16.1 & & 17.9 & \\
\hline No one to turn to for support (yes) & 18.5 & $<0.001$ & 16.0 & $<0.001$ & 21.7 & $<0.001$ \\
\hline No one to turn to for support (no) & 15.9 & & 15.1 & & 16.5 & \\
\hline \multicolumn{7}{|l|}{ Other cultural variable } \\
\hline \multicolumn{7}{|l|}{ Cultural engagement } \\
\hline Clothing or footwear (yes) & 17.0 & $<0.001$ & 17.0 & $<0.001$ & 17.1 & 0.002 \\
\hline Clothing or footwear (no) & 16.0 & & 15.1 & & 16.7 & \\
\hline Art craft (yes) & 17.4 & $<0.001$ & 16.6 & $<0.001$ & 17.9 & $<0.001$ \\
\hline Art craft (no) & 15.5 & & 14.8 & & 16.2 & \\
\hline Hunting/fishing/trapping (yes) & 15.6 & $<0.001$ & 14.9 & $<0.001$ & 16.7 & 0.7 \\
\hline Hunting/fishing/trapping (no) & 16.3 & & 15.5 & & 16.8 & \\
\hline Plant gathering (yes) & 16.5 & $<0.001$ & 15.3 & 0.05 & 17.4 & $<0.001$ \\
\hline Plant gathering (no) & 15.9 & & 15.1 & & 16.5 & \\
\hline \multicolumn{7}{|l|}{ Language } \\
\hline Speak Indigenous language (yes) & 15.9 & 0.04 & 14.6 & $<0.001$ & 16.7 & 0.8 \\
\hline Speak Indigenous language (no) & 16.1 & & 15.3 & & 16.8 & \\
\hline \multicolumn{7}{|l|}{ Residential school variable } \\
\hline Not attended & 15.7 & $<0.001$ & 15.0 & $<0.001$ & 16.3 & $<0.001$ \\
\hline Any family member attended & 16.7 & & 15.6 & & 17.5 & \\
\hline Missing & 17.1 & & 15.8 & & 18.2 & \\
\hline \multicolumn{7}{|l|}{ Geographical variable } \\
\hline \multicolumn{7}{|l|}{ Urbanicity } \\
\hline Rural & 15.0 & $<0.001$ & 14.7 & $<0.001$ & 15.3 & $<0.001$ \\
\hline Small population centre & 15.4 & & 14.7 & & 16.0 & \\
\hline Medium population centre & 16.8 & & 15.1 & & 17.8 & \\
\hline Urban & 16.7 & & 15.7 & & 17.5 & \\
\hline \multicolumn{7}{|l|}{ Regions } \\
\hline Atlantic provinces & 15.3 & $<0.001$ & 14.2 & $<0.001$ & 16.1 & $<0.001$ \\
\hline Quebec & 15.5 & & 15.2 & & 15.8 & \\
\hline Ontario & 16.6 & & 15.5 & & 17.5 & \\
\hline Manitoba & 16.4 & & 15.5 & & 17.1 & \\
\hline Saskatchewan & 15.5 & & 14.8 & & 16.0 & \\
\hline Alberta & 16.0 & & 15.0 & & 16.6 & \\
\hline British Columbia & 16.2 & & 15.2 & & 16.9 & \\
\hline Territories & 15.2 & & 14.5 & & 15.7 & \\
\hline
\end{tabular}


Table 3 (part 1 of 2): Prevalence of lifetime suicidal ideations and suicide attempts among Indigenous peoples living off-reserve $(n=14410)$

\begin{tabular}{|c|c|c|c|c|c|c|c|c|c|c|c|c|}
\hline \multirow[b]{3}{*}{ Characteristic } & \multicolumn{6}{|c|}{ Lifetime suicidal ideation } & \multicolumn{6}{|c|}{ Lifetime suicide attempt } \\
\hline & \multicolumn{2}{|c|}{ Total } & \multicolumn{2}{|c|}{ Men } & \multicolumn{2}{|c|}{ Women } & \multicolumn{2}{|c|}{ Total } & \multicolumn{2}{|c|}{ Men } & \multicolumn{2}{|c|}{ Women } \\
\hline & $\%$ & $p$ value & $\%$ & $p$ value & $\%$ & $p$ value & $\%$ & $p$ value & $\%$ & $p$ value & $\%$ & $p$ value \\
\hline Total prevalence & 19.4 & & 16.1 & & 21.9 & & 2.2 & & 2.0 & & 2.3 & \\
\hline \multicolumn{13}{|l|}{ Demographic variable } \\
\hline \multicolumn{13}{|l|}{ Sex } \\
\hline Male & 16.1 & $<0.001$ & & & & & 2.0 & 0.002 & & & & \\
\hline Female & 21.9 & & & & & & 2.3 & & & & & \\
\hline \multicolumn{13}{|l|}{ Marital status } \\
\hline Married & 15.4 & $<0.001$ & 12.9 & $<0.001$ & 17.6 & $<0.001$ & 1.4 & $<0.001$ & NR & & 1.4 & $<0.001$ \\
\hline Divorced or widowed & 27.8 & & 22.9 & & 29.6 & & 2.0 & & NR & & 2.2 & \\
\hline Single & 22.2 & & 19.8 & & 24.1 & & 3.7 & & NR & & 4.0 & \\
\hline \multicolumn{13}{|l|}{ Cultural group } \\
\hline Métis & 17.5 & $<0.001$ & 13.8 & $<0.001$ & 20.5 & $<0.001$ & 1.7 & $<0.001$ & 1.2 & $<0.001$ & 2.2 & $<0.001$ \\
\hline Status First Nations & 21.3 & & 18.8 & & 23.5 & & 2.4 & & 2.5 & & 2.4 & \\
\hline Non-status First Nations & 19.6 & & 16.2 & & 22.2 & & 2.7 & & 3.5 & & 2.0 & \\
\hline Inuit & 22.7 & & 20.7 & & 24.3 & & 3.2 & & 1.6 & & 4.5 & \\
\hline \multicolumn{13}{|l|}{ Socioeconomic variable } \\
\hline \multicolumn{13}{|l|}{ Education level } \\
\hline$\leq$ Grade 8 & 14.8 & $<0.001$ & 15.1 & $<0.001$ & 14.6 & $<0.001$ & 1.7 & $<0.001$ & 2.5 & $<0.001$ & 0.9 & $<0.001$ \\
\hline Grades $9-10$ & 23.3 & & 21.1 & & 25.2 & & 4.1 & & 3.3 & & 4.8 & \\
\hline Grade 11-secondary completed & 18.5 & & 15.3 & & 21.2 & & 2.1 & & 2.0 & & 2.2 & \\
\hline Some postsecondary & 20.7 & & 18.9 & & 22.2 & & 2.3 & & 1.9 & & 2.7 & \\
\hline Postsecondary degree/diploma & 19.0 & & 14.1 & & 22.4 & & 1.8 & & 1.6 & & 1.9 & \\
\hline \multicolumn{13}{|l|}{ Employment status } \\
\hline Employed & 17.5 & $<0.001$ & 15.5 & $<0.001$ & 19.1 & $<0.001$ & 1.6 & $<0.001$ & 1.5 & $<0.001$ & 1.6 & $<0.001$ \\
\hline Not in the labour force & 23.4 & & 18.9 & & 25.6 & & 3.5 & & 3.7 & & 3.4 & \\
\hline Unemployed & 21.3 & & 15.0 & & 27.2 & & 2.7 & & 2.0 & & 3.3 & \\
\hline \multicolumn{13}{|l|}{ Household crowding } \\
\hline Rooms per capita $\leq 2$ & 21.4 & $<0.001$ & 17.8 & $<0.001$ & 24.0 & $<0.001$ & 2.6 & $<0.001$ & NR & & 2.8 & $<0.001$ \\
\hline Rooms per capita $>2$ to 4 & 17.4 & & 13.8 & & 20.1 & & 1.8 & & NR & & 1.7 & \\
\hline Rooms per capita > 4 & 16.1 & & 15.5 & & 16.6 & & 1.6 & & NR & & 1.6 & \\
\hline \multicolumn{13}{|l|}{ Housing maintenance } \\
\hline Regular & 17 & $<0.001$ & 14.5 & $<0.001$ & 18.9 & $<0.001$ & 1.6 & $<0.001$ & 1.4 & $<0.001$ & 1.7 & $<0.001$ \\
\hline Minor & 20.0 & & 17.1 & & 22.5 & & 2.4 & & 2.4 & & 2.4 & \\
\hline Major & 27.4 & & 20.3 & & 32.1 & & 4.0 & & 3.2 & & 4.5 & \\
\hline \multicolumn{13}{|l|}{ Food security } \\
\hline High & 14.8 & $<0.001$ & 12.5 & $<0.001$ & 16.7 & $<0.001$ & 1.2 & $<0.001$ & 1.4 & $<0.001$ & 1.1 & $<0.001$ \\
\hline Low & 34.4 & & 30.6 & & 36.9 & & 3.8 & & 2.2 & & 5.0 & \\
\hline Very low & 44.4 & & 43.1 & & 45.0 & & 9.3 & & 9.9 & & 9.0 & \\
\hline Behavioural variable & & & & & & & & & & & & \\
\hline Drinking habit & & & & & & & & & & & & \\
\hline Regular drinker (yes) & 16.7 & $<0.001$ & 14.5 & $<0.001$ & 18.9 & $<0.001$ & 2.2 & 0.8 & 2.0 & 0.9 & 2.4 & 0.3 \\
\hline Regular drinker (no) & 22.9 & & 19.2 & & 24.9 & & 2.2 & & 2.0 & & 2.3 & \\
\hline Smoking habit & & & & & & & & & & & & \\
\hline Daily tobacco use (yes) & 27.8 & $<0.001$ & 25.1 & $<0.001$ & 29.8 & $<0.001$ & 3.8 & $<0.001$ & 3.6 & $<0.001$ & 4.0 & $<0.001$ \\
\hline Daily tobacco use (no) & 15.6 & & 12.1 & & 18.3 & & 1.4 & & 1.3 & & 1.6 & \\
\hline
\end{tabular}


Table 3 (part 2 of 2): Prevalence of lifetime suicidal ideations and suicide attempts among Indigenous peoples living off-reserve $(n=14410)$

\section{Social connectedness variable}

Strong extended family tie (yes)

Strong extended family tie (no)

No one to turn to for support (yes)

Lifetime suicidal ideation

Lifetime suicide attempt

No one to turn to for support (no)

\begin{tabular}{|c|c|c|c|c|c|c|c|c|c|c|c|}
\hline \multicolumn{2}{|c|}{ Total } & \multicolumn{2}{|c|}{ Men } & \multicolumn{2}{|c|}{ Women } & \multicolumn{2}{|c|}{ Total } & \multicolumn{2}{|c|}{ Men } & \multicolumn{2}{|c|}{ Women } \\
\hline$\%$ & $p$ value & $\%$ & $p$ value & $\%$ & $p$ value & $\%$ & $p$ value & $\%$ & $p$ value & $\%$ & $p$ value \\
\hline
\end{tabular}

$\begin{array}{lllllllllllll}15.7 & <0.001 & 11.2 & <0.001 & 18.6 & <0.001 & 1.2 & <0.001 & 1.0 & <0.001 & 1.3 & <0.001 \\ 23.2 & & 20.4 & & 25.8 & & 3.2 & & 2.8 & & 3.6 & \\ 27.0 & <0.001 & 20.6 & <0.001 & 35.1 & <0.001 & 5.7 & <0.001 & 4.2 & <0.001 & 7.5 & <0.001 \\ 19.0 & & 15.8 & & 21.4 & & 2.0 & & 1.8 & & 2.1 & \end{array}$

Other cultural variable

Cultural engagement

Clothing or footwear (yes)
Clothing or footwear (no)
Art craft (yes)
Art craft (no)
Hunting/fishing/trapping (yes)
Hunting/fishing/trapping (no)
Plant gathering (yes)
Plant gathering (no)
Language
Speak Indigenous language (yes)
Speak Indigenous language (no)

\begin{tabular}{lllllllllllll}
30.2 & $<0.001$ & 33.2 & $<0.001$ & 29.8 & $<0.001$ & 3.2 & $<0.001$ & 6.6 & $<0.001$ & 2.7 & 0.002 \\
18.2 & & 15.5 & & 20.5 & & 2.1 & & 1.8 & & 2.2 & \\
\hline 27.6 & $<0.001$ & 23.2 & $<0.001$ & 29.8 & $<0.001$ & 2.8 & $<0.001$ & 1.9 & 0.5 & 3.2 & $<0.001$ \\
16.1 & & 14.1 & & 17.9 & & 1.9 & & 2.0 & & 1.9 & \\
17.6 & $<0.001$ & 14.9 & $<0.001$ & 21.5 & 0.2 & 2.2 & 0.7 & 2.3 & $<0.001$ & 2.1 & 0.006 \\
\hline 20.4 & & 17.3 & & 22.0 & & 2.2 & & 1.7 & & 2.4 & \\
17.7 & $<0.001$ & 15.4 & $<0.001$ & 19.6 & $<0.001$ & 2.1 & 0.001 & 2.1 & 0.06 & 2.1 & $<0.001$ \\
23.1 & & 18.0 & & 26.4 & & 2.4 & & 1.7 & & 2.9 & \\
& & & & & & & & & & & \\
\hline 20.8 & $<0.001$ & 17.6 & $<0.001$ & 22.7 & 0.06 & 2.5 & $<0.001$ & 1.7 & 0.01 & 3.1 & $<0.001$ \\
19.2 & & 15.9 & & 21.8 & & 2.1 & & 2.0 & & 2.2 &
\end{tabular}

\section{Residential school variable}

Not attended

Any family member attended

\begin{tabular}{|c|c|c|c|c|c|c|c|c|c|c|}
\hline 17.0 & $<0.001$ & 14.0 & $<0.001$ & 19.3 & $<0.001$ & 1.6 & $<0.001$ & 1.7 & $<0.001$ & 1.6 \\
\hline 24.3 & & 20.2 & & 26.9 & & 3.4 & & 3.0 & & 3.7 \\
\hline 26.6 & & 24.0 & & 28.4 & & 3.5 & & 1.7 & & 4.8 \\
\hline
\end{tabular}

24.0

28.4

3.5

Geographical variable

Urbanicity

\begin{tabular}{|c|c|c|c|c|c|c|c|c|c|c|c|c|}
\hline Rural & 15.5 & $<0.001$ & 13.7 & $<0.001$ & 17.1 & $<0.001$ & 1.8 & $<0.001$ & 2.1 & $<0.001$ & 1.5 & $<0.001$ \\
\hline Small population centre & 17.5 & & 15.3 & & 19.3 & & 1.9 & & 2.3 & & 1.6 & \\
\hline Medium population centre & 22.1 & & 16.6 & & 25.6 & & 3.4 & & 2.9 & & 3.7 & \\
\hline Urban & 21.5 & & 17.7 & & 24.3 & & 2.2 & & 1.5 & & 2.7 & \\
\hline \multicolumn{13}{|l|}{ Region } \\
\hline Atlantic provinces & 12.0 & $<0.001$ & 8.5 & $<0.001$ & 14.4 & $<0.001$ & 0.8 & $<0.001$ & NR & & 1.3 & $<0.001$ \\
\hline Quebec & 22.3 & & 22.2 & & 22.4 & & 2.4 & & NR & & 1.8 & \\
\hline Ontario & 20.0 & & 16.2 & & 22.9 & & 2.5 & & NR & & 2.6 & \\
\hline Manitoba & 15.6 & & 13.6 & & 17.1 & & 2.5 & & NR & & 1.9 & \\
\hline Saskatchewan & 17.3 & & 12.2 & & 20.8 & & 1.5 & & NR & & 2.0 & \\
\hline Alberta & 21.6 & & 16.4 & & 25.5 & & 2.1 & & NR & & 3.3 & \\
\hline British Columbia & 21.3 & & 17.3 & & 24.4 & & 2.3 & & NR & & 2.0 & \\
\hline Territories & 21.2 & & 21.8 & & 20.8 & & 2.7 & & NR & & 3.5 & \\
\hline
\end{tabular}

Note: NR = not reported. ${ }^{*}$

*Values are not reported per the data disclosure policy of Statistics Canada's Research Data Centres for small cell sizes (fewer than 10 unweighted individuals with the outcome variable in 1 of the subgroups of each categorical variables). 
Table 4 (part 1 of 2): Psychological distress and suicidal behaviours among Indigenous peoples living off-reserve, by income quartile $(n=14410)$

\begin{tabular}{|c|c|c|c|c|c|}
\hline \multirow[b]{2}{*}{ Variable } & \multicolumn{4}{|c|}{$\begin{array}{c}\text { Mean score on the K10 or prevalence of suicidal behaviours, } \\
\text { by income quartile }\end{array}$} & \multirow[b]{2}{*}{$C$ or $C_{n}(95 \% \mathrm{Cl})^{\star}$} \\
\hline & $\begin{array}{c}\text { First } \\
\text { (lowest) }\end{array}$ & Second & Third & $\begin{array}{l}\text { Fourth } \\
\text { (highest) }\end{array}$ & \\
\hline $\begin{array}{l}\text { Psychological distress (mean score } \\
\text { on the K10) }\end{array}$ & & & & & c \\
\hline \multicolumn{6}{|l|}{ Total (both sexes) } \\
\hline All Indigenous groups & 18.4 & 16.1 & 15.4 & 14.3 & $-0.054(-0.057$ to -0.050$)$ \\
\hline Métis & 18.2 & 15.9 & 15.2 & 14.3 & $-0.054(-0.060$ to -0.048$)$ \\
\hline Status First Nations & 18.1 & 16.0 & 15.6 & 14.5 & $-0.069(-0.082$ to -0.057$)$ \\
\hline Non-status First Nations & 20.2 & 16.3 & 15.9 & 14.6 & $-0.049(-0.056$ to -0.042$)$ \\
\hline Inuit & 17.1 & 15.4 & 14.9 & 14.2 & $-0.039(-0.048$ to -0.030$)$ \\
\hline \multicolumn{6}{|l|}{ Men } \\
\hline All Indigenous groups & 16.9 & 15.3 & 14.8 & 13.7 & $-0.046(-0.051$ to -0.040$)$ \\
\hline Métis & 17.1 & 15.2 & 14.3 & 13.6 & $-0.052(-0.060$ to -0.044$)$ \\
\hline Status First Nations & 16.3 & 15.6 & 15.3 & 13.9 & $-0.059(-0.076$ to -0.041$)$ \\
\hline Non-status First Nations & 18.4 & 15.5 & 14.0 & 14.3 & $-0.032(-0.042$ to -0.022$)$ \\
\hline Inuit & 15.3 & 15.3 & 14.5 & 13.2 & $-0.032(-0.045$ to -0.019$)$ \\
\hline \multicolumn{6}{|l|}{ Women } \\
\hline All Indigenous groups & 19.4 & 16.6 & 16.0 & 14.9 & $-0.055(-0.060$ to -0.050$)$ \\
\hline Métis & 19.0 & 16.3 & 16.1 & 15.0 & $-0.050(-0.058$ to -0.042$)$ \\
\hline Status First Nations & 19.3 & 16.7 & 15.6 & 14.8 & $-0.073(-0.090$ to -0.057$)$ \\
\hline Non-status First Nations & 21.1 & 17.6 & 17.1 & 14.8 & $-0.057(-0.066$ to -0.048$)$ \\
\hline Inuit & 18.4 & 15.6 & 15.3 & 14.8 & $-0.044(-0.057$ to -0.031$)$ \\
\hline Lifetime suicidal ideation (prevalence, \%) & & & & & $C_{n}$ \\
\hline \multicolumn{6}{|l|}{ Total (both sexes) } \\
\hline All Indigenous groups & 27.7 & 21.4 & 15.2 & 13.1 & $-0.218(-0.242$ to -0.194$)$ \\
\hline Métis & 27.3 & 16.5 & 13.8 & 12.1 & $-0.236(-0.274$ to -0.198$)$ \\
\hline Status First Nations & 27.0 & 24.3 & 18.0 & 16.0 & $-0.269(-0.343$ to -0.196$)$ \\
\hline Non-status First Nations & 30.8 & 22.0 & 13.5 & 12.0 & $-0.165(-0.205$ to -0.125$)$ \\
\hline Inuit & 31.5 & 21.5 & 20.2 & 17.8 & $-0.153(-0.208$ to -0.098$)$ \\
\hline \multicolumn{6}{|l|}{ Men } \\
\hline All Indigenous groups & 24.0 & 16.6 & 13.7 & 10.1 & $-0.229(-0.267$ to -0.191$)$ \\
\hline Métis & 23.2 & 10.8 & 11.9 & 9.1 & $-0.237(-0.298$ to -0.176$)$ \\
\hline Status First Nations & 23.1 & 20.1 & 18.2 & 13.9 & $-0.378(-0.496$ to -0.260$)$ \\
\hline Non-status First Nations & 31.1 & 15.1 & 10.5 & 7.9 & $-0.141(-0.205$ to -0.077$)$ \\
\hline Inuit & 26.7 & 23.1 & 17.2 & 15.8 & $-0.151(-0.236$ to -0.066$)$ \\
\hline \multicolumn{6}{|l|}{ Women } \\
\hline All Indigenous groups & 29.4 & 25.1 & 17.6 & 15.2 & $-0.196(-0.227$ to -0.165$)$ \\
\hline Métis & 30.6 & 20.2 & 15.5 & 15.7 & $-0.215(-0.264$ to -0.166$)$ \\
\hline Status First Nations & 30.2 & 26.1 & 20.8 & 15.1 & $-0.197(-0.291$ to -0.103$)$ \\
\hline Non-status First Nations & 30.9 & 24.9 & 17.6 & 15.2 & $-0.167(-0.219$ to -0.115$)$ \\
\hline Inuit & 34.9 & 20.9 & 21.5 & 20.2 & $-0.153(-0.226$ to -0.080$)$ \\
\hline
\end{tabular}


Table 4 (part 2 of 2): Psychological distress and suicidal behaviours among Indigenous peoples living off-reserve, by income quartile $(n=14410)$

\begin{tabular}{|c|c|c|c|c|c|}
\hline \multirow[b]{2}{*}{ Variable } & \multicolumn{4}{|c|}{$\begin{array}{c}\text { Mean score on the K10 or prevalence of suicidal behaviours, } \\
\text { by income quartile }\end{array}$} & \multirow[b]{2}{*}{$C$ or $C_{n}(95 \% \mathrm{CI})^{\star}$} \\
\hline & $\begin{array}{c}\text { First } \\
\text { (lowest) }\end{array}$ & Second & Third & $\begin{array}{l}\text { Fourth } \\
\text { (highest) }\end{array}$ & \\
\hline Lifetime suicide attempt (prevalence, \%) & & & & & $C_{n}$ \\
\hline \multicolumn{6}{|l|}{ Total (both sexes) } \\
\hline All Indigenous groups & 4.5 & 1.8 & 1.3 & 1.1 & $-0.327(-0.391$ to -0.263$)$ \\
\hline Métis & 3.7 & 1.2 & 1.3 & 0.7 & $-0.354(-0.467$ to -0.241$)$ \\
\hline Status First Nations & 4.3 & 2.6 & 1.5 & 1.3 & $-0.382(-0.565$ to -0.199$)$ \\
\hline Non-status First Nations & NR & NR & NR & NR & $-0.280(-0.388$ to -0.172$)$ \\
\hline Inuit & 4.5 & 3.0 & 3.1 & 2.3 & $-0.171(-0.303$ to -0.039$)$ \\
\hline \multicolumn{6}{|l|}{ Men } \\
\hline All Indigenous groups & 4.8 & 1.5 & 1.1 & 0.5 & $-0.462(-0.562$ to -0.362$)$ \\
\hline Métis & NR & NR & NR & NR & $-0.506(-0.706$ to -0.306$)$ \\
\hline Status First Nations & NR & NR & NR & NR & $-0.629(-0.868$ to -0.390$)$ \\
\hline Non-status First Nations & NR & NR & NR & NR & $-0.325(-0.486$ to -0.164$)$ \\
\hline Inuit & NR & NR & NR & NR & $-0.341(-0.619$ to -0.063$)$ \\
\hline \multicolumn{6}{|l|}{ Women } \\
\hline All Indigenous groups & 4.1 & 1.8 & 1.5 & 1.8 & $-0.236(-0.320$ to -0.152$)$ \\
\hline Métis & 4.4 & 1.0 & 1.7 & 1.4 & $-0.269(-0.407$ to -0.131$)$ \\
\hline Status First Nations & 3.6 & 3.0 & 1.6 & 1.3 & $-0.128(-0.408$ to 0.152$)$ \\
\hline Non-status First Nations & NR & NR & NR & NR & $-0.242(-0.387$ to -0.097$)$ \\
\hline Inuit & NR & NR & NR & NR & $-0.126(-0.278$ to 0.026$)$ \\
\hline $\begin{array}{l}\text { Note: } C=\text { concentration index, } \mathrm{Cl}=\text { confidence interval, } \\
{ }^{*} \mathrm{~A} \text { negative value of the } C \text { indicates that the health out } \\
\text { tValues are not reported per the data disclosure policy } \\
\text { variable in } 1 \text { of the income quartiles). }\end{array}$ & $\begin{array}{l}\text { malized con } \\
\text { disproportio } \\
\text { stics Canada' }\end{array}$ & $\begin{array}{l}\text { ndex, K10 } \\
\text { entrated } \\
\text { Data Cen }\end{array}$ & $\begin{array}{l}\text { essler } \\
\text { with | } \\
\text { | cell si }\end{array}$ & $\begin{array}{l}\text { Distress Sca } \\
\text { n } 10 \text { unweig }\end{array}$ & $\begin{array}{l}\text { not reported. } \dagger \\
\text { dividuals with the outcome }\end{array}$ \\
\hline
\end{tabular}

3.2\%; women: $20.2 \%$ ) of the income-related inequalities in psychological distress, lifetime suicidal ideation and lifetime suicide attempt, respectively. The negative contribution of food insecurity to the $C$ and $C_{n}$ suggests that food insecurity increases the concentration of mental health outcomes among low-income Indigenous peoples in Canada.

Moreover, the income variable itself contributed negatively to income-related inequalities in psychological distress and lifetime suicidal ideation for men and women. Income accounted for 13.7\% (men: $12.9 \%$; women: $14.0 \%$ ) of income-related inequalities in psychological distress, $18.2 \%$ (men: $20.1 \%$; women: $17.6 \%$ ) of those in lifetime suicidal ideation, and $7.9 \%$ of those in suicide attempt among men. Employment status explained 13.5\% (men: $11.1 \%$; women: $14.9 \%$ ) of income-related inequalities in psychological distress and $7.2 \%$ (men: $3.1 \%$; women: $7.9 \%$ ) of income-related inequalities in suicide attempt. Unexplained variables accounted for a residual 5.5\% (men: $16.3 \%$; women: $-0.5 \%$ ) of income-related inequalities in psychological distress, but $20.3 \%$ (men: $31.7 \%$; women: $12.8 \%$ ) of incomerelated inequalities in suicidal ideation and 63.0\% (men: $79.7 \%$; women: $63.7 \%$ ) of income-related inequalities in suicide attempt.

\section{Interpretation}

We showed high prevalence rates of psychological distress, lifetime suicidal ideation and suicide attempt among Indigenous peoples living off-reserve in Canada in 2012. Income-related inequality in psychological distress was higher among women than men, while income-related inequalities in suicidal behaviours were higher among men. Across the 4 groups compared, income-related inequalities in mental health outcomes were greater among status First Nations men and Métis women.

The results of our study are consistent with those of an analysis in Saskatchewan, which showed higher household income to be a protective factor against lifetime suicidal ideation among Indigenous peoples. ${ }^{49}$ Our results are also consistent with the findings of previous studies examining associations between income and mental health outcomes among non-Indigenous populations. A 2005 study involving 200 low-income African Americans found that life hassles (defined as work and time pressure), social and cultural difficulties, finances, and social acceptability and victimization, were all independently significant risk factors for suicide attempt. ${ }^{50} \mathrm{~A}$ case-control study in New York 
Table 5: Absolute $(\%)^{*}$ contribution of determinants to income-related inequalities in psychological distress and suicidal behaviours among Indigenous peoples living off-reserve $(n=14410)$

\begin{tabular}{|c|c|c|c|c|c|c|c|c|c|}
\hline \multirow[b]{2}{*}{ Determinants $\dagger$} & \multicolumn{3}{|c|}{ Psychological distress } & \multicolumn{3}{|c|}{ Lifetime suicidal ideation } & \multicolumn{3}{|c|}{ Lifetime suicide attempt } \\
\hline & Total & Men & Women & Total & Men & Women & Total & Men & Women \\
\hline Demographic & $0.0039(7.2)$ & $-0.0011(2.5)$ & $-0.0023(4.1)$ & $-0.0202(9.3)$ & $-0.0098(4.3)$ & $-0.0196(10.0)$ & $-0.0095(2.9)$ & $-0.0035(0.8)$ & $-0.0140(5.9)$ \\
\hline Cultural group & $0014(-2.6)$ & $0.0009(-1.9)$ & $0.0016(-2.9)$ & $-0.0039(1.8)$ & $-0.0056(2.4)$ & $-0.0018(0.9)$ & $0.0008(-0.3)$ & $-0.0046(1.0)$ & $0.0037(-1.5)$ \\
\hline Income & .0074 (13.7) & $-0.0059(12.9)$ & $-0.0077(14.0)$ & $-0.0397(18.2)$ & $-0.0461(20.1)$ & $-0.0344(17.6)$ & $-0.0255(7.8)$ & $-0.0367(7.9)$ & $0.0102(-4.3)$ \\
\hline Education & $0.0019(3.5)$ & $-0.0014(3.0)$ & $-0.0024(4.3)$ & $0.0147(-6.7)$ & $0.0077(-3.4)$ & $0.0220(-11.2)$ & $0.0069(-2.1)$ & $-0.0001(0.2)$ & $0.0107(-4.5)$ \\
\hline Employment status & $.0073(13.5)$ & $-0.0051(11.1)$ & $-0.0082(14.9)$ & $-0.0024(1.1)$ & $0.0107(-4.7)$ & $-0.0127(6.5)$ & $-0.0235(7.2)$ & $-0.0141(3.1)$ & $-0.0186(7.9)$ \\
\hline $\begin{array}{l}\text { Household crowding } \\
\text { and housing condition }\end{array}$ & $0.0016(2.9)$ & $-0.0008(1.7)$ & $-0.0021(3.9)$ & $-0.0089(4.1)$ & $-0.0029(1.2)$ & $-0.0136(7.0)$ & $\begin{array}{c}-0.00004 \\
(0.01)\end{array}$ & $0.0019(-0.4)$ & $0.0010(-0.4)$ \\
\hline Food security & $-0.0217(40.2)$ & $-0.0176(38.4)$ & $-0.0236(42.9)$ & $-0.0581(26.7)$ & $-0.0489(21.4)$ & $-0.0632(32.2)$ & $-0.0437(13.4)$ & $-0.0148(3.2)$ & $-0.0477(20.2)$ \\
\hline Behavioural factors & $-0.0049(9.0)$ & $-0.0061(13.2)$ & $-0.0042(7.7)$ & $-0.0342(15.7)$ & $-0.0417(18.2)$ & $-0.0270(13.8)$ & $-0.0119(3.6)$ & $-0.0170(3.7)$ & $-0.0075(3.2)$ \\
\hline $\begin{array}{l}\text { Social connectedness } \\
\text { factors }\end{array}$ & $0.0017(3.1)$ & $-0.0010(2.1)$ & $-0.0029(5.2)$ & $-0.0044(2.0)$ & $-0.0050(2.2)$ & $-0.0060(3.0)$ & $-0.0081(2.5)$ & $-0.0055(1.2)$ & $-0.0092(3.9)$ \\
\hline Other cultural factors & $-0.0003(0.6)$ & $-0.0001(0.2)$ & $-0.0001(0.2)$ & $-0.0075(3.4)$ & $-0.0056(2.5)$ & $-0.0016(0.8)$ & $0.0014(-0.4)$ & $0.0115(-2.5)$ & $-0.0033(1.4)$ \\
\hline Residential school & $-0.0010(1.8)$ & $-0.0009(2.0)$ & $-0.0008(1.5)$ & $-0.0073(3.3)$ & $-0.0080(3.5)$ & $-0.0066(3.4)$ & $-0.0081(2.5)$ & $-0.0036(0.8)$ & $-0.0079(3.3)$ \\
\hline Geographical & $-0.0009(1.6)$ & $0.0007(-1.4)$ & $-0.0025(4.6)$ & $-0.0017(0.8)$ & $-0.0011(0.5)$ & $-0.0064(3.2)$ & $0.0002(-0.1)$ & $-0.0070(1.5)$ & $-0.0032(1.4)$ \\
\hline esidual & $-0.0030(5.5)$ & $-0.0075(16.3)$ & $0.0002(-0.5)$ & $-0.0443(20.3)$ & $-0.0727(31.7)$ & $-0.0251(12.8)$ & $-0.2059(63.0)$ & $-0.3684(79.7)$ & $-0.1503(63.7)$ \\
\hline$c$ & -0.0536 & -0.0455 & -0.0548 & -0.2181 & -0.2294 & -0.1960 & -0.3272 & -0.4623 & -0.2358 \\
\hline
\end{tabular}

Note: $C=$ concentration index, $C_{n}=$ normalized concentration index.

*Percentage contributions in parentheses were calculated by dividing the corresponding contribution value by the $C / C_{n}$. Detailed results of the decomposition analyses can be found in Appendices 1D, $1 \mathrm{E}$ and $1 \mathrm{~F}$.

†For variables within each determinant, see subsection "Explanatory variables" in the Methods.

City suggested that suicide decedents were more likely to be younger and reside in communities with high income inequality and low income per capita. ${ }^{51}$

A complex interplay of biological, social and cultural factors contribute to mental health problems. ${ }^{52}$ Within this complex interplay, food insecurity is recognized as one of the main factors contributing to the poor mental health outcomes within Indigenous and non-Indigenous populations. ${ }^{53-64}$ Data from the 2012 Canadian Community Health Survey showed that $28.2 \%$ of off-reserve Indigenous households experienced some form of food insecurity in the past year, compared with $12.6 \%$ within the overall Canadian population. ${ }^{65}$ The decomposition results indicated that food insecurity made the largest contribution to the concentration of psychological distress and suicidal behaviours among low-income Indigenous peoples in Canada. Greater food insecurity increases probabilities of poor mental health outcomes, and food insecurity is more prevalent among the poor. The contribution of food insecurity to income-related inequalities in the 3 mental health outcomes was greater among women than men.

Based on our results, addressing food insecurity among lowincome Indigenous peoples living off-reserve may potentially reduce a substantial proportion of the observed income-related inequalities in mental health outcomes. Our findings also point to the importance of household income and employment status as contributors to income-related inequalities in the 3 mental health outcomes. The contribution of employment is important because, compared with employed Indigenous peoples, Indigenous peoples who are not in the labour force or are unemployed had higher probabilities of having mental health problems and were generally poorer.

\section{Limitations}

Our analyses have several limitations. First, the Aboriginal Peoples Survey does not collect information from individuals living in institutions (e.g., prisons and hospitals) and other collective dwellings (e.g., shelters, rooming houses and group homes), where a disproportionate number of Indigenous people reside. This exclusion omits many Indigenous peoples known to be at high-risk for adverse mental health problems. ${ }^{66,67}$ Second, we used the lifetime time frame to examine suicidal behaviours in the study to ensure a large enough number of respondents with outcome variables for the analyses. It would be ideal for future studies to have concurrent measures of outcome and explanatory variables. Third, some of the explanatory factors in the decomposition analysis are likely to be endogenous and may lead to unknown bias in the study. For example, endogeneity in the case of smoking behaviour can be due to the impossibility of distinguishing causality (i.e., whether smoking leads to a mental health issue or mental health issues leads to smoking). Fourth, 
owing to the unavailability of these data in the Aboriginal Peoples Survey, our analyses included limited historical, cultural, and contemporary factors that are likely important determinants of Indigenous health, including the diversity of nations and cultures that exist within First Nations, Inuit and Métis peoples. Fifth, the contributions of the residual component to income-related inequalities in lifetime suicide ideation and attempt were substantial. This suggests that other factors not included in our models affect socioeconomic inequalities in mental health outcomes among Indigenous peoples. This could also be due, in part, to the less-than-optimal specification of the explanatory variables included in our models. Finally, because we used a survey of living individuals, we did not consider completed suicides, which excluded the most severe mental health outcome in our analyses.

\section{Conclusion}

We found persistent and substantial income-related inequalities in psychological distress and suicidal behaviours among Indigenous peoples living off-reserve in Canada. Indigenous peoples in Canada have high rates of mental health problems and experience some of the greatest inequalities in social determinants of health. 4,19,20 Our findings underscore the need for greater policy attention to socioeconomic inequalities in mental health within Indigenous peoples as well as inequalities in mental health between Indigenous and non-Indigenous people. To address mental health issues faced by Indigenous peoples, policies must address a broader array of social determinants, such as food insecurity, income and employment status.

\section{References}

1. Dohrenwend BP, Shrout PE, Egri G, et al. Nonspecific psychological distress and other dimensions of psychopathology. Measures for use in the general population. Arch Gen Psychiatry 1980;37:1229-36.

2. McLoughlin AB, Gould MS, Malone KM. Global trends in teenage suicide: 20032014. QJM 2015;108:765-80.

3. Hatcher S, Crawford A, Coupe N. Preventing suicide in indigenous communities. Curr Opin Psychiatry 2017;30:21-5.

4. Kirmayer L, Brass GM, Holton T, et al. Suicide among Aboriginal people in Canada. Ottawa: Aboriginal Healing Foundation; 2007.

5. Acting on what we know: Preventing youth suicide in First Nations. Ottawa: Health Canada, Advisory Group on Suicide Prevention; 2003.

6. Tjepkema M, Wilkins R, Senécal S, et al. Mortality of Métis and registered Indian adults in Canada: an 11-year follow-up study. Health Rep 2009;20: 31-51.

7. The human face of mental health and mental illness in Canada 2006. Ottawa: Public Health Agency of Canada; 2006.

8. Kral MJ. Suicide and suicide prevention among Inuit in Canada. Can J Psychiatry 2016;61:688-95.

9. Peters PA. Causes and contributions to differences in life expectancy for Inuit Nunangat and Canada, 1994-2003. Int J Circumpolar Health 2010;69:38-49.

10. Malchy B, Enns MW, Young TK, et al. Suicide among Manitoba's aboriginal people, 1988 to 1994 . CMAJ 1997;156:1133-8.

11. Tjepkema M, Wilkins R, Pennock J, et al. Potential years of life lost at ages 25 to 74 among Status Indians, 1991 to 2001. Health Rep 2011;22:25-36.

12. Kumar MB. Aboriginal Peoples Survey, 2012: lifetime suicidal thoughts among First Nations living off reserve, Métis and Inuit aged 26 to 59: prevalence and associated characteristics. Ottawa: Statistics Canada; 2016. Cat no 89-653-X.

13. Doyle K. Measuring cultural appropriateness of mental health services for Australian Aboriginal peoples in rural and remote Western Australia: a client/ clinician's journey. Int J Cult Ment Health 2012;5:40-53.

14. Kirmayer LJ, Valaskakis GG. Healing traditions: the mental health of Aboriginal peoples in Canada. Vancouver: University of British Columbia Press; 2009.
15. Kral MJ, Idlout L, Minore JB, et al. Unikkaartuit: meanings of well-being, unhappiness, health, and community change among Inuit in Nunavut, Canada. Am J Community Psychol 2011;48:426-38.

16. Marrone S. Understanding barriers to health care: a review of disparities in health care services among indigenous populations. Int J Circumpolar Health 2007;66:188-98.

17. Waldram JB, Herring DA, Young TK. Aboriginal health in Canada: historical, cultural, and epidemiological perspectives. Toronto: University of Toronto Press; 2006.

18. The health status of Canada's First Nations, Métis, and Inuit peoples. Toronto: Health Council of Canada; 2005.

19. Adelson N. The embodiment of inequity: health disparities in aboriginal Canada. Can J Public Health 2005;96:S45-61.

20. Pendakur K, Pendakur R. Aboriginal income disparity in Canada. Can Public Policy 2011;37:61-83.

21. Mitrou F, Cooke M, Lawrence D, et al. Gaps in Indigenous disadvantage not closing: a census cohort study of social determinants of health in Australia, Canada, and New Zealand from 1981-2006. BMC Public Health 2014;14:201.

22. Hajizadeh M, Hu M, Bombay A, et al. Socioeconomic inequalities in health among Indigenous Peoples living off-reserve in Canada: trends and determinants. Health Policy 2018;122:854-65.

23. Hajizadeh M, Mitnitski A, Rockwood K. Socioeconomic gradient in health in Canada: Is the gap widening or narrowing? Health Policy 2016;120:1040-50.

24. Mangalore R, Knapp M, Jenkins R. Income-related inequality in mental health in Britain: the concentration index approach. Psychol Med 2007;37:1037-45.

25. Data products, 2016 census. Ottawa: Statistics Canada; 2019. Available: www12. statcan.gc.ca/census-recensement/2016/dp-pd/index-Eng.cfm (accessed 2019 Feb. 10).

26. Cloutier E, Langlet É. Aboriginal Peoples Survey, 2012: concepts and methods guide. Ottawa: Statistics Canada; 2014. Cat no 89-653-X.

27. Budinski R, Langlet E. Aboriginal Peoples Survey 2012: user's guide to the public use microdata file (PUMF). Ottawa: Statistics Canada; 2015.

28. Kessler RC, Andrews G, Colpe LJ, et al. Short screening scales to monitor population prevalences and trends in non-specific psychological distress. Psychol Med 2002;32:959-76.

29. Bougie E, Arim RG, Kohen DE, et al. Validation of the 10-item Kessler psychological distress scale (K10) in the 2012 Aboriginal Peoples Survey. Health Rep 2016;27:3-10.

30. Kessler RC, Barker PR, Colpe LJ, et al. Screening for serious mental illness in the general population. Arch Gen Psychiatry 2003;60:184-9.

31. Furukawa TA, Kessler RC, Slade T, et al. The performance of the K6 and K10 screening scales for psychological distress in the Australian National Survey of Mental Health and Well-Being. Psychol Med 2003;33:357-62.

32. Growing unequal?: income distribution and poverty in OECD countries. Paris: Organisation for Economic Co-operation and Development Publishing; 2008.

33. McGrail KM, Van Doorslaer E, Ross NA, et al. Income-related health inequalities in Canada and the United States: a decomposition analysis. Am J Public Health 2009;99:1856-63.

34. Cai J, Coyte PC, Zhao H. Determinants of and socio-economic disparities in self-rated health in China. Int J Equity Health 2017;16:7.

35. Marmot M, Wilkinson RG. The social determinants of health. Oxford (UK): Oxford University Press; 2006

36. Reading $\mathrm{CL}$, Wein F. Health inequalities and social determinants of Aboriginal Peoples' health. Prince George (BC): National Collaborating Centre for Aboriginal Health; 2009.

37. Peter T, Roberts LW, Buzdugan R. Suicidal ideation among Canadian youth: a multivariate analysis. Arch Suicide Res 2008;12:263-75.

38. McNamara BJ, Banks E, Gubhaju L, et al. Factors relating to high psychological distress in Indigenous Australians and their contribution to Indigenous-nonIndigenous disparities. Aust N Z J Public Health 2018;42:145-52.

39. Anderson T. The social determinants of higher mental distress among Inuit. Ottawa: Statistics Canada; 2015. Cat no 89-653-X.

40. Bombay A, Matheson $\mathrm{K}$, Anisman $\mathrm{H}$. The intergenerational effects of Indian residential schools: implications for the concept of historical trauma. Transcult Psychiatry 2014;51:320-38.

41. Hackett C, Feeny D, Tompa E. Canada's residential school system: measuring the intergenerational impact of familial attendance on health and mental health outcomes. J Epidemiol Community Health 2016;70:1096-105.

42. Wagstaff A, Paci P, van Doorslaer E. On the measurement of inequalities in health. Soc Sci Med 1991;33:545-57.

43. Quantitative techniques for health equity analysis - technical note \#7. The Concentration Index. Geneva: World Bank; 2019. Available: http://citeseerx.ist.psu. edu/viewdoc/summary?doi=10.1.1.634.5222 (accessed 2019 Feb. 10). 
44. O'Donnell O, van Doorslaer E, Wagstaff A, et al. Analyzing health equity using Household Survey data - a guide to techniques and their implementation. Geneva: The World Bank; 2008.

45. Wagstaff $A$. The bounds of the concentration index when the variable of interest is binary, with an application to immunization inequality. Health Econ 2005; 14:429-32.

46. Wagstaff A, van Doorslaer E, Watanabe N. On decomposing the causes of health sector inequalities with an application to malnutrition inequalities in Vietnam. J Econom 2003;112:207-23.

47. The Research Data Centres Information and Technical Bulletin. Ottawa: Statistics Canada; 2014. Cat no 12-002-X - no 2014001.

48. StataCorp. Stata Statistical Software: Release 14. 2015

49. Lemstra M, Neudorf C, Mackenbach J, et al. Suicidal ideation: the role of economic and Aboriginal cultural status after multivariate adjustment. Can J Psychiatry 2009;54:589-95.

50. Kaslow NJ, Sherry A, Bethea K, et al. Social risk and protective factors for suicide attempts in low income African American men and women. Suicide Life Threat Behav 2005;35:400-12.

51. Miller JR, Piper TM, Ahern J, et al. Income inequality and risk of suicide in New York City neighborhoods: a multilevel case-control study. Suicide Life Threat Behav 2005;35:448-59.

52. A report of mental illness in Canada. Ottawa: Public Health Agency of Canada; 2002.

53. Runnels VE, Kristjansson E, Calhoun M. An investigation of adults' everyday experiences and effects of food insecurity in an urban area in Canada. Can J Commun Ment Health 2011;30:157-72.

54. Hamelin A-M, Beaudry M, Habicht J-P. Characterization of household food insecurity in Québec: food and feelings. Soc Sci Med 2002;54:119-32.

55. Fuller-Thomson E, Nimigon J. Factors associated with depression among individuals with chronic fatigue syndrome: findings from a nationally representative survey. Fam Pract 2008;25:414-22.

56. Jessiman-Perreault G, McIntyre L. The household food insecurity gradient and potential reductions in adverse population mental health outcomes in Canadian adults. SSM Popul Health 2017;3:464-72.
57. Davison KM, Marshall-Fabien GL, Tecson A. Association of moderate and severe food insecurity with suicidal ideation in adults: national survey data from three Canadian provinces. Soc Psychiatry Psychiatr Epidemiol 2015;50 963-72.

58. Muldoon KA, Duff PK, Fielden S, et al. Food insufficiency is associated with psychiatric morbidity in a nationally representative study of mental illness among food insecure Canadians. Soc Psychiatry Psychiatr Epidemiol 2013;48:795-803.

59. Whitaker RC, Phillips SM, Orzol SM. Food insecurity and the risks of depression and anxiety in mothers and behavior problems in their preschool-aged children. Pediatrics 2006;118:e859-68.

60. Tarasuk V, Mitchell A, McLaren L, et al. Chronic physical and mental health conditions among adults may increase vulnerability to household food insecurity. J Nutr 2013;143:1785-93.

61. Siefert K, Heflin CM, Corcoran ME, et al. Food insufficiency and physical and mental health in a longitudinal survey of welfare recipients. J Health Soc Behav 2004;45:171-86.

62. Pryor L, Lioret S, van der Waerden J, et al. Food insecurity and mental health problems among a community sample of young adults. Soc Psychiatry Psychiatr Epidemiol 2016;51:1073-81.

63. Leung CW, Epel ES, Willett WC, et al. Household food insecurity is positively associated with depression among low-income supplemental nutrition assistance program participants and income-eligible nonparticipants. J Nutr 2015;145: 622-7.

64. Heflin CM, Siefert K, Williams DR. Food insufficiency and women's mental health findings from a 3-year panel of welfare recipients. Soc Sci Med 2005;61:1971-82.

65. Sunderland M, Mahoney A, Andrews G. Investigating the factor structure of the Kessler Psychological Distress Scale in community and clinical samples of the Australian population. J Psychopathol Behav Assess 2012;34:253-9.

66. Kouyoumdjian F, Schuler A, Matheson FI, et al. Health status of prisoners in Canada: narrative review. Can Fam Physician 2016;62:215-22.

67. Hwang SW, Wilkins R, Tjepkema M, et al. Mortality among residents of shelters, rooming houses, and hotels in Canada: 11-year follow-up study. BMJ 2009;339: b4036.
Competing interests: Mohammad Hajizadeh, Amy Bombay and Yukiko Asada report grants from the Nova Scotia Health Research Foundation.

This article has been peer reviewed.

Affiliations: School of Health Administration (Hajizadeh); Department of Psychiatry and School of Nursing (Bombay); Department of Community Health and Epidemiology (Asada), Dalhousie University, Halifax, NS

Contributors: All authors contributed to the conception and design of the study. Mohammad Hajizadeh performed the statistical analysis and all authors interpreted the results. Mohammad Hajizadeh drafted the manuscript, and Amy Bombay and Yukiko Asada contributed to drafting and revisions. All authors read and approved the final version of the manuscript and agreed to be accountable for all aspects of the work.

Funding: Funding for this research was provided by the Nova Scotia Health Research Foundation Establishment Grant program (grant no. 1017).

Acknowledgements: The analyses presented in this paper were conducted at the Statistics Canada's Atlantic Research Data Centre at Dalhousie University, which is part of the Canadian Research Data Centre Network. The authors thank the Canadian Research Data Centre Network for facilitating the access to the Aboriginal Peoples Survey and the Atlantic Research Data Centre analyst Heather Hobson for her support and assistance. The authors also thank participants at the 52nd Annual Conference of the Canadian Economics Association, the 12th European Conference on Health Economics and the 2018 Canadian Association for Health Services and Policy Research for their comments and suggestions.

Accepted: Feb. 28, 2019

Correspondence to: Mohammad Hajizadeh, m.hajizadeh@dal.ca 